\title{
Same-day genomic and epigenomic diagnosis of brain tumors using real-time nanopore sequencing
}

\author{
Philipp Euskirchen ${ }^{1,2,3}$ (1) - Franck Bielle ${ }^{1,4,5} \cdot$ Karim Labreche $^{1,6} \cdot$ Wigard P. Kloosterman $^{7} \cdot$ Shai Rosenberg $^{1} \cdot$ \\ Mailys Daniau ${ }^{1}$ - Charlotte Schmitt $^{1} \cdot$ Julien Masliah-Planchon $^{8} \cdot$ Franck Bourdeaut $^{10}$ - Caroline Dehais $^{9}$ • \\ Yannick Marie $^{1} \cdot$ Jean-Yves Delattre $^{1,9} \cdot$ Ahmed Idbaih $^{1,9}$
}

Received: 3 March 2017 / Revised: 7 June 2017 / Accepted: 10 June 2017 / Published online: 21 June 2017

(c) The Author(s) 2017. This article is an open access publication

\begin{abstract}
Molecular classification of cancer has entered clinical routine to inform diagnosis, prognosis, and treatment decisions. At the same time, new tumor entities have been identified that cannot be defined histologically. For central nervous system tumors, the current World Health Organization classification explicitly demands molecular testing, e.g., for $1 \mathrm{p} / 19 \mathrm{q}$-codeletion or IDH mutations, to make an integrated histomolecular diagnosis. However, a plethora of sophisticated technologies is currently needed to assess different genomic and epigenomic alterations and turnaround times are in the range of weeks, which makes standardized and widespread implementation difficult and hinders timely decision making. Here, we explored the potential of a pocket-size nanopore sequencing device for multimodal and rapid molecular diagnostics of cancer. Low-pass whole genome sequencing was used to simultaneously generate copy number $(\mathrm{CN})$ and methylation profiles from native tumor DNA in the same sequencing run.
\end{abstract}

Electronic supplementary material The online version of this article (doi:10.1007/s00401-017-1743-5) contains supplementary material, which is available to authorized users.

Philipp Euskirchen

philipp.euskirchen@ charite.de

$\triangle$ Ahmed Idbaih

ahmed.idbaih@aphp.fr

1 Inserm U 1127, CNRS UMR 7225, Sorbonne Universités, UPMC Univ Paris 06 UMR S 1127, Institut du Cerveau et de la Moelle épinière (ICM), Paris, France

2 Department of Neurology, Charité-Universitätsmedizin Berlin, Berlin, Germany

3 Berlin Institute of Health (BIH), Berlin, Germany

4 Service de Neuropathologie, AP-HP, Hôpitaux Universitaires La Pitié Salpêtrière-Charles Foix, Paris, France
Single nucleotide variants in IDH1, IDH2, TP53, H3F3A, and the TERT promoter region were identified using deep amplicon sequencing. Nanopore sequencing yielded $\sim 0.1 \mathrm{X}$ genome coverage within $6 \mathrm{~h}$ and resulting $\mathrm{CN}$ and epigenetic profiles correlated well with matched microarray data. Diagnostically relevant alterations, such as $1 \mathrm{p} / 19 \mathrm{q}$ codeletion, and focal amplifications could be recapitulated. Using ad hoc random forests, we could perform supervised pancancer classification to distinguish gliomas, medulloblastomas, and brain metastases of different primary sites. Single nucleotide variants in $I D H 1, I D H 2$, and $H 3 F 3 A$ were identified using deep amplicon sequencing within minutes of sequencing. Detection of TP53 and TERT promoter mutations shows that sequencing of entire genes and GCrich regions is feasible. Nanopore sequencing allows sameday detection of structural variants, point mutations, and methylation profiling using a single device with negligible capital cost. It outperforms hybridization-based and current sequencing technologies with respect to time to diagnosis and required laboratory equipment and expertise, aiming to

5 OncoNeuroTek, Paris, France

6 Division of Genetics and Epidemiology, The Institute of Cancer Research, Sutton, Surrey SM2 5NG, UK

7 Division of Biomedical Genetics, Center for Molecular Medicine, Department of Genetics, University Medical Center Utrecht, Utrecht, The Netherlands

8 Department of Genetics, Institut Curie, PSL Research University, Paris, France

9 Service de Neurologie, AP-HP, Hôpitaux Universitaires La Pitié Salpêtrière-Charles Foix, 2-Mazarin, Paris, France

10 Laboratory of Translational Research in Pediatric Oncology, Institut Curie, PSL Research University, Paris, France 
make precision medicine possible for every cancer patient, even in resource-restricted settings.

Keywords Nanopore sequencing · Brain tumor - Glioma · Whole genome sequencing $\cdot$ Epigenomics $\cdot$ Molecular neuropathology

\section{Introduction}

Histomolecular classification of brain tumors has entered clinical routine diagnostics as the current World Health Organization (WHO) classification explicitly demands histological findings to be refined by molecular testing [20]. Thus, pathologists rely on timely and accurate molecular testing to make an integrated diagnosis using both in situ methods and genetic information. However, high turnaround time of current implementations delays integrated diagnosis by weeks. In addition, targeted next-generation sequencing panels, microarray-based analysis of copy number $(\mathrm{CN})$, and epigenetic alterations all provide highquality data and aid in the diagnosis and therapeutic management of patients (i.e., stratification or identification of actionable targets or inclusion in clinical trials), but their high capital cost, demanding workflows and need for highly skilled personnel hinder their widespread use. Here, we demonstrate that real-time molecular genomics using nanopore sequencing is both fast and reliable to aid diagnosing cancer by unsupervised classification of $\mathrm{CN}$ and methylation profiles.

Nanopore sequencing interprets changes in ionic currents observed when single DNA molecules pass through a nanometer-size protein pore. This has led to the development of handheld size devices that allow sequencing outside of classical laboratory settings and even in the field [27]. While overall throughput currently lacks behind other deep sequencing technologies, nanopores allow read analysis in real-time and selective sequencing [19], both of which allow rapid generation of data. In addition, nanopores are able to discriminate not only the nucleotides of a strand of DNA but also single base modifications such as 5-methylation of cytosine [29, 35]. This allows concurrent analysis of sequence identity and methylation using native DNA.

\section{Materials and methods}

\section{Experimental design}

We performed a retrospective observational study for molecular characterization of diagnostically relevant genetic alterations using nanopore sequencing. Patients were recruited at the Pitié-Salpêtrière university hospital and have given informed consent for research use of tumor material, including genotyping. All tumor samples have been molecularly characterized previously using short-read exome sequencing, Sanger sequencing, SNP array, and/or genome-wide methylation microarray $[14,30]$.

\section{Nanopore whole genome sequencing}

DNA quality of fresh-frozen tumor tissue was determined using NanoDrop (Thermo Fisher Scientific) and samples were quantified using a QuantiFluor dsDNA assay (Promega, Madison, WI, USA). For whole genome sequencing, libraries were prepared using Rapid 1D Sequencing Kit (SQK-RAD001, SQK-RAD002, or SQK-RBK001, Oxford Nanopore Technologies, UK) following the manufacturer's instructions. Briefly, $200 \mathrm{ng}$ of tumor DNA was fragmented using a transposase and subjected to adapter ligation. Sequencing was performed using R9 or R9.4 flow cells on a MinION Mk 1B device (Oxford Nanopore) with the MinKNOW software (versions 1.0.5-1.5.12), respectively. For samples run with R9.4 sequencing chemistry, basecalling was performed using Albacore 1.1.0 (Oxford Nanopore). For R9 chemistries, online EPI2ME basecalling (Metrichor Ltd, Oxford, UK) was performed.

Template reads were exported as FASTA using nanopolish or poretools version 0.6 [18] and aligned to the hg19 human reference genome using BWA MEM 0.7.12 with the " $-\mathrm{x}$ ont2d" option [17]. Due to compatibility issues of data generated with R9 chemistries, only samples with R9.4 flow cells were used for copy number analysis and methylation-based classification.

\section{Copy number analysis}

For copy number analysis, the QDNAseq package version 1.8.0 [33] and R/Bioconductor, version 3.3, were used. Reads with a minimum mapping quality of 20 were sorted into $1000 \mathrm{kbp}$ bins. Bins with missing reference sequence were excluded from analysis. To account for region- and technology-specific artifacts, public nanopore WGS data for the NA12878 human reference genome were processed identically and subtracted from the normalized tumor sample bin counts. Circular binary segmentation was performed as implemented in the DNAcopy package requiring an alpha value $<0.05$ to accept change points. Arm-level copy number calls were made by calculating the segment length weighted mean log ratio per chromosome arm.

\section{Methylation analysis}

To identify 5-methylation of cytosines, we used a recently published algorithm based on a hidden Markov model 
which has been trained using in vitro methylated $E$. coli DNA [35]. Training models for R9 sequencing chemistries were kindly provided by Jared Simpson. We modified the original implementation of nanopolish 0.6.0 to allow methylation calling from different basecalling groups. For classification, the subset of $\mathrm{CpG}$ sites overlapping with sites covered by Illumina 450K BeadChip microarrays was used. Beta values in the training set were dichotomized using a cut-off value of 0.6 .

\section{Structural variant detection}

For detection of structural variants in amplified regions, we aligned nanopore FASTQ files from sample 3427T to the human reference genome, build GRCh37, using LAST (version 744) with settings: $-Q 0$. The last-train function was used with 1000 nanopore reads ( 10 million bases) as input to adapt the alignment scoring parameters $(-p)$ for error-prone nanopore reads. LAST alignment files (MAF) were converted to BAM files using the maf-convert function. BAM files were used as input for NanoSV [36] (available at https://github.com/mroosmalen/nanosv) with default settings.

\section{Amplicon sequencing}

Amplicons were designed to cover one or multiple exons of canonical transcripts of IDH1,IDH2, TP53, H3F3A, and the TERT promoter region. Oligonucleotide primers (Thermo Fisher Scientific) were then designed using Primer3 with the following non-default parameters $\left(T_{\min }\right.$ $59{ }^{\circ} \mathrm{C}, T_{\text {opt }} 60{ }^{\circ} \mathrm{C}, T_{\max } 61{ }^{\circ} \mathrm{C}$, and maximum mononucleotide repeat length $=3$ ) to yield product sizes of 4892902 bp (Table S1).

$25 \mathrm{ng}$ of genomic DNA was amplified using $0.02 \mathrm{U} /$ $\mu \mathrm{l}$ Q5 polymerase (New England Biolabs, Ipswich, MA, USA), $200 \mu \mathrm{M}$ dNTPs, $500 \mathrm{nM}$ forward and reverse primers, and Q5 reaction buffer with high GC enhancer in a total reaction volume of $20 \mu \mathrm{l}$. Thermal cycling was performed as follows: $98{ }^{\circ} \mathrm{C}$ initial denaturation for $2 \mathrm{~min}$, followed by 30 cycles of denaturation at $98{ }^{\circ} \mathrm{C}$ for $10 \mathrm{~s}$, annealing at $65^{\circ} \mathrm{C}$ for $20 \mathrm{~s}$ and extension at $72{ }^{\circ} \mathrm{C}$ for $90 \mathrm{~s}$, as well as a final extension at $72{ }^{\circ} \mathrm{C}$ for $2 \mathrm{~min}$. Amplicons were analyzed using a Caliper LabChip GX DNA 5K assay (Perkin Elmer, Waltham, MA, USA). PCR products were purified using NucleoFast 96 PCR plates (Macherey-Nagel, Düren, Germany).

For amplicon sequencing, Ligation Sequencing Kit 1D (SQK-LSK108, Oxford) was used following the manufacturer's protocol. Briefly, $1 \mu \mathrm{g}$ of pooled amplicon DNA was subjected to end repair and dA-tailing. $250 \mathrm{ng}$ of endrepaired DNA (equivalent to $0.2 \mathrm{pmol}$ of $2 \mathrm{kbp}$ fragments) was then used as input for adapter ligation. For real-time monitoring of sequencing depth, reads were streamed to the BWA aligner using npReader [6] with jHDF5 2.11.0 and coverage was calculated using BEDTools [28]. For variant calling, reads were realigned on the event level and variants called using VarScan 2.4.3 [15]. Variants were annotated using SnpEff version 4.3i [9] and ExAC release 0.3.1 germline variants [16] before filtering for coding or hotspot mutations with a minimum mutant allele frequency $>0.2$.

\section{Microarray methylation profiling}

Samples for Illumina Infinium BeadChip $450 \mathrm{~K}$ profiling were prepared as described before [14]. Briefly, $500 \mathrm{ng}$ of DNA was subjected to bisulfite conversion. Hybridization and imaging were performed by IntegraGen (Evry, France). Raw IDAT files were preprocessed using the GenomeStudio software (Illumina, San Diego, CA, USA). Processed methylation data from previously characterized samples [14] were retrieved via ArrayExpress (accession E-MTAB-3903). Beta values were used for all the subsequent analysis steps.

\section{Statistics}

All data analysis was done using $\mathrm{R} /$ Bioconductor version 3.3 [13]. Hierarchical clustering was used for arranging probes in the depicted classification training set. Random forest classification as implemented in the $\mathrm{R} /$ randomForest package, version 4.6-12, was run with default parameters. Sequence concordance was calculated using the Genome Analysis Toolkit's Genotype Concordance tool, version 3.7 [21].

\section{Data and material availability}

Raw sequencing data are available via the European Genome-phenome Archive (accession EGAS00001002213). Microarray-based methylome data are available at ArrayExpress (E-MTAB-5797). TCGA data were retrieved from the UCSC Cancer Browser [11] or the TCGA FireBrowse website (http://www.firebrowse.org). Pipelines, scripts, and supplementary data to reproduce all results presented in this work are available at https://gitlab.com/pesk/glioma. nano-seq.

\section{Results}

To meet the needs of the WHO 2016 classification of CNS tumors, we designed 1-day workflows for $\mathrm{CN}$, methylation, and point mutation profiling using nanopore sequencing (Fig. 1a). We first subjected tumor DNA from molecularly well-characterized brain tumors $[14,30]$ to 


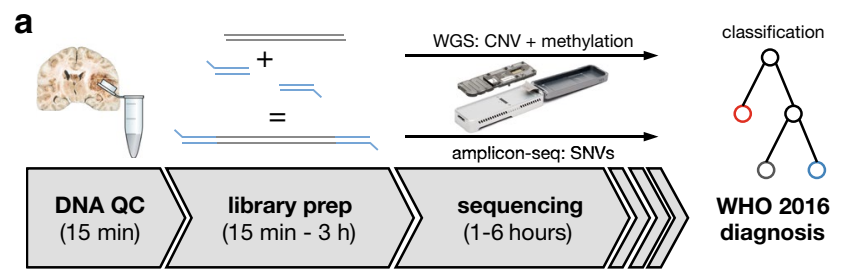

d
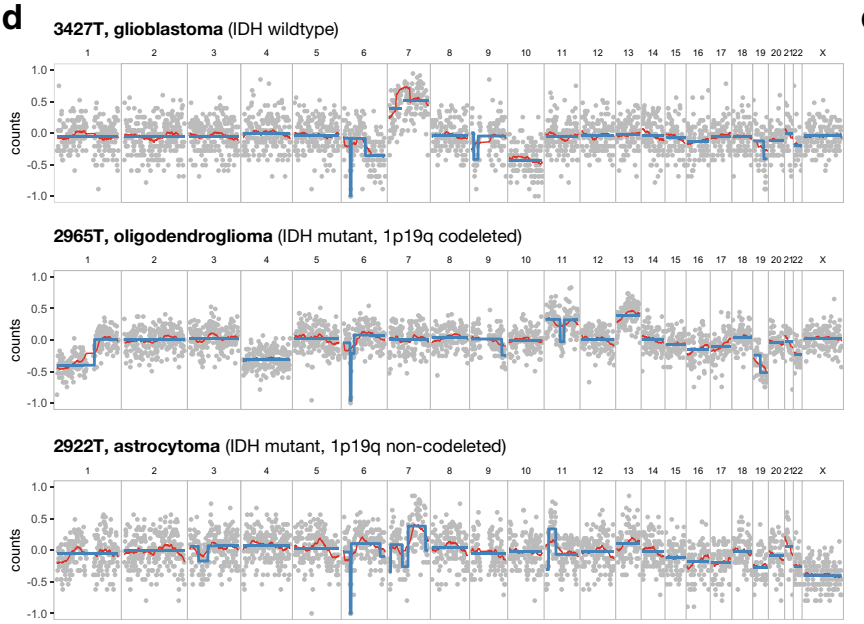

Fig. 1 Copy number profiling using nanopore low-pass whole genome sequencing. a Same-day workflows to simultaneously characterize copy number variation (CNV) and methylation profiles or single nucleotide variants, respectively. Tumor DNA is subjected to quality control (QC), and then, $250 \mathrm{ng}$ input material is used for library preparation for either whole genome sequencing (WGS) or PCR-based deep amplicon sequencing. b Representative read length distribution of mapped reads. Note $\log$ scale on $X$ axis. $\mathbf{c}$ Representa-

low-pass whole genome sequencing (WGS) using a commercially available, handheld size nanopore sequencing device. With the aim of widespread implementation in routine diagnostics in mind, we used a transposon-based library preparation kit, which reduces sample preparation time to less than hour. In a cohort of 28 patients (Table 1), low-pass WGS for $6 \mathrm{~h}$ performed yielded a mean mapped read depth from $<0.01 \mathrm{X}$ to $0.24 \mathrm{X}$ (Table S1), depending on the sequencing chemistry and input DNA fragment size. Nanopores decipher DNA sequence of single molecules as they present to the pore, generating long reads of variable length, whose distribution is determined by DNA extraction and fragmentation method. We observed typical mean read lengths around $2 \mathrm{~kb}$ (Fig. 1b). As library preparation does not involve PCR amplification, no GC bias is introduced and the GC content distribution of the reads resembles closely that of the human reference genome (Fig. 1c).
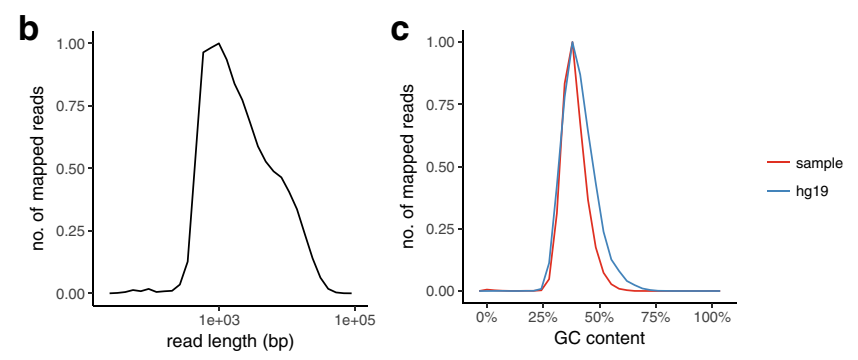

e

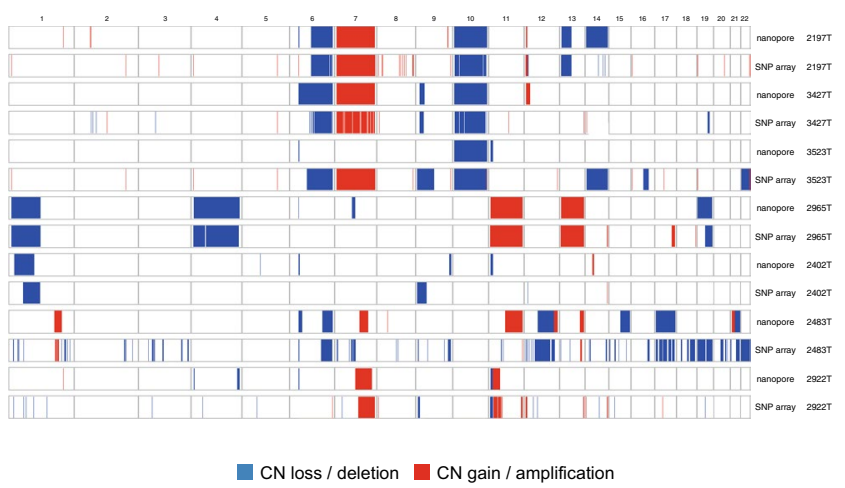

tive distribution of GC content of reads in comparison with the hg19 human reference genome. A randomly drawn subsample of the entire reference genome split into $1000 \mathrm{bp}$ fragments is shown. d Copy number profile showing $\log _{2}$ transformed, normalized read counts per $1000 \mathrm{kbp}$ window (grey) with running mean (red) and segmentation results (blue). e Comparison of nanopore WGS with matched SNP arrays. Heatmaps indicate copy number calls (losses and deletions in blue, and gains and amplifications in red) across the genome

\section{Copy number profiling}

We then used WGS data to generate $\mathrm{CN}$ profiles. Reads were counted in $1000 \mathrm{~kb}$ windows, normalized and subjected to circular binary segmentation (Fig. 1c). No correction of GC bias or mappability is necessary for nanopore reads; however, the long reads cause alignment artifacts with current reference genomes in regions with repetitive sequence such as centromeres. Still, the resulting CN profiles closely resembled matched SNP array-based profiles (Fig. 1d). Importantly, codeletion of chromosome 1p/19q as a diagnostic criterion for oligodendrogliomas implemented in the 2016 WHO classification of CNS tumors was detected in three out of four affected samples (Fig. S1). The remaining sample did not yield sufficient read depth $(<0.01)$ due to low input DNA quality (Table S1). Highlevel focal amplifications of EGFR, PDGFRA, and CDK4 were detected in affected glioblastoma samples (Table 1). 
Table 1 Clinical characteristics of patients in study

\begin{tabular}{|c|c|c|c|c|c|c|}
\hline ID & Age at diagnosis & Sex & $\begin{array}{l}\text { WHO } 2016 \text { integrated } \\
\text { diagnosis }\end{array}$ & $\begin{array}{l}\text { Nanopore sequencing } \\
\text { performed }\end{array}$ & $\begin{array}{l}\text { Nanopore methylation- } \\
\text { based classification }\end{array}$ & $\begin{array}{l}\text { Key alterations identified } \\
\text { by nanopore sequencing }\end{array}$ \\
\hline $3523 \mathrm{~T}$ & 70 & $\mathrm{~F}$ & $\begin{array}{l}\text { Glioblastoma, } \\
\text { IDH-wildtype }\end{array}$ & WGS, amplicon & Not classifiable & pTERT C228T \\
\hline $2197 \mathrm{~T}$ & 58 & $\mathrm{~F}$ & $\begin{array}{l}\text { Glioblastoma, } \\
\text { IDH-wildtype }\end{array}$ & WGS, amplicon & Glioma, IDH-wildtype & $\begin{array}{l}\text { TP53 p.S241F, pTERT } \\
\text { C228T }\end{array}$ \\
\hline $3427 \mathrm{~T}$ & 72 & $\mathrm{~F}$ & $\begin{array}{l}\text { Glioblastoma, } \\
\text { IDH-wildtype }\end{array}$ & WGS, amplicon & Glioma, IDH-wildtype & $\begin{array}{l}\text { pTERT C228T, } \\
\text { CDKN2A }^{\text {loss }}, \text { EGFR }^{\text {amp }}\end{array}$ \\
\hline $2402 \mathrm{~T}$ & 58 & M & $\begin{array}{l}\text { Anaplastic oligodendro- } \\
\text { glioma, IDH-mutant, } \\
\text { and } 1 \mathrm{p} / 19 \mathrm{q} \text {-codeleted }\end{array}$ & WGS, amplicon & Not classifiable & $\begin{array}{l}\text { IDH1 p.R132H, 1p/19q } \\
\text { codeletion, pTERT } \\
\text { C228T }\end{array}$ \\
\hline $2965 \mathrm{~T}$ & 29 & $\mathrm{~F}$ & $\begin{array}{l}\text { Anaplastic oligodendro- } \\
\text { glioma, IDH-mutant } \\
\text { and } 1 \mathrm{p} / 19 \mathrm{q} \text {-codeleted }\end{array}$ & WGS, amplicon & Glioma, IDH-mutant & $\begin{array}{l}\text { IDH1 p.R132H, 1p/19q } \\
\text { codeletion, pTERT } \\
\text { C228T }\end{array}$ \\
\hline $2483 \mathrm{~T}$ & 51 & $\mathrm{~F}$ & $\begin{array}{l}\text { Anaplastic astrocytoma, } \\
\text { IDH-mutant }\end{array}$ & WGS, amplicon & Glioma, IDH-mutant & $\begin{array}{l}\text { IDH1 p.R132C } \\
\text { TP53 p.R273C, p.R282Q }\end{array}$ \\
\hline $2922 \mathrm{~T}$ & 44 & M & $\begin{array}{l}\text { Diffuse astrocytoma, } \\
\text { IDH-mutant }\end{array}$ & WGS & Glioma, IDH-mutant & N/D \\
\hline $6228 \mathrm{~T}$ & 33 & $\mathrm{~F}$ & $\begin{array}{l}\text { Diffuse midline glioma, } \\
\text { H3.3 K27M-mutant }\end{array}$ & WGS, amplicon & Classifiable & PDGFRA $^{\text {amp }}$ \\
\hline $5337 \mathrm{~T}$ & 21 & M & Glioma H3.3 G34R & WGS, amplicon & Glioma IDH-wildtype & $\begin{array}{l}\text { H3F3A G34R, CDK4 } 4^{\text {amp }}, \\
\text { PDGFRA }^{\text {amp }}\end{array}$ \\
\hline $8347 \mathrm{~T}$ & 28 & M & $\begin{array}{l}\text { Desmoplastic/nodular } \\
\text { medulloblastoma, } \\
\text { SHH-activated and } \\
\text { TP53 wild type }\end{array}$ & Amplicon & N/D & pTERT C228T \\
\hline $8372 \mathrm{~T}$ & 25 & M & $\begin{array}{l}\text { Classic medulloblas- } \\
\text { toma, non-WNT/ } \\
\text { non-SHH }\end{array}$ & WGS, amplicon & $\begin{array}{l}\text { Medulloblastoma, } \\
\text { group } 4\end{array}$ & pTERT C228T \\
\hline MB683 & 7 & $\mathrm{~F}$ & $\begin{array}{l}\text { Classic medulloblas- } \\
\text { toma, WNT-activated }\end{array}$ & WGS, amplicon & $\begin{array}{l}\text { Medulloblastoma, } \\
\text { WNT-activated }\end{array}$ & chr6 loss \\
\hline $8137 \mathrm{~T}$ & 48 & M & $\begin{array}{l}\text { Anaplastic oligodendro- } \\
\text { glioma, IDH-mutant } \\
\text { and } 1 \mathrm{p} / 19 \mathrm{q} \text {-codeleted }\end{array}$ & WGS, amplicon & Glioma, IDH-mutant & $\begin{array}{l}\text { IDH2 p.R172 W, 1p/19q } \\
\text { codeletion, pTERT } \\
\text { C228T }\end{array}$ \\
\hline $8146 \mathrm{~T}$ & N/A & $\mathrm{F}$ & $\begin{array}{l}\text { Anaplastic oligodendro- } \\
\text { glioma, IDH-mutant } \\
\text { and } 1 \mathrm{p} / 19 \mathrm{q} \text {-codeleted }\end{array}$ & WGS, amplicon & Glioma, IDH-mutant & pTERT C228T \\
\hline $7382 \mathrm{~T}$ & 76 & $\mathrm{~F}$ & $\begin{array}{l}\text { Glioblastoma, } \\
\text { IDH-wildtype }\end{array}$ & WGS, amplicon & Glioma, IDH-wildtype & $\begin{array}{l}\text { pTERT C228T, } \\
\text { PDGFRA }^{\text {amp }} \\
\text { TP53 p.V197M }\end{array}$ \\
\hline $7455 \mathrm{~T}$ & 45 & M & $\begin{array}{l}\text { Glioblastoma, } \\
\text { IDH-wildtype }\end{array}$ & WGS, amplicon & Glioma, IDH-wildtype & pTERT C228T \\
\hline $8355 \mathrm{~T}$ & 56 & M & $\begin{array}{l}\text { Glioblastoma, } \\
\text { IDH-wildtype }\end{array}$ & WGS & Not classifiable & N/D \\
\hline $8356 \mathrm{~T}$ & 73 & $\mathrm{~F}$ & $\begin{array}{l}\text { Breast adenocarcinoma, } \\
\text { GFAP }+, \text { S100+ }\end{array}$ & WGS & Breast cancer & N/D \\
\hline $8357 \mathrm{~T}$ & 79 & M & $\begin{array}{l}\text { Neuro-endrocrine (pros- } \\
\text { tate adeno) carcinoma, } \\
\text { TTF1+ }\end{array}$ & WGS & Lung cancer & N/D \\
\hline $8358 \mathrm{~T}$ & 63 & $\mathrm{~F}$ & Lung adenocarcinoma & WGS & Lung cancer & N/D \\
\hline $8359 \mathrm{~T}$ & 51 & M & $\begin{array}{l}\text { Bladder urothelial } \\
\text { carcinoma }\end{array}$ & WGS, amplicon & Not classifiable & TP53 p.R280 K \\
\hline $8360 \mathrm{~T}$ & 65 & $\mathrm{~F}$ & Lung adenocarcinoma & Amplicon & N/D & TP53 p.I195T \\
\hline 4596T FFPE & 44 & $\mathrm{~F}$ & $\begin{array}{l}\text { Anaplastic oligodendro- } \\
\text { glioma, IDH-mutant } \\
\text { and } 1 \mathrm{p} / 19 \mathrm{q} \text {-codeleted }\end{array}$ & WGS, amplicon & Not classifiable & pTERT C228T \\
\hline
\end{tabular}


Table 1 continued

\begin{tabular}{|c|c|c|c|c|c|c|}
\hline ID & Age at diagnosis & Sex & $\begin{array}{l}\text { WHO } 2016 \text { integrated } \\
\text { diagnosis }\end{array}$ & $\begin{array}{l}\text { Nanopore sequencing } \\
\text { performed }\end{array}$ & $\begin{array}{l}\text { Nanopore methylation- } \\
\text { based classification }\end{array}$ & $\begin{array}{l}\text { Key alterations identified } \\
\text { by nanopore sequencing }\end{array}$ \\
\hline 5539T FFPE & 28 & M & $\begin{array}{l}\text { Anaplastic astrocytoma, } \\
\text { IDH-mutant }\end{array}$ & Amplicon & N/D & pTERT C228T $\mathrm{T}^{\mathrm{II}}$ \\
\hline $3718 \mathrm{~T}$ & 78 & $\mathrm{~F}$ & $\begin{array}{l}\text { Glioblastoma, } \\
\text { IDH-wildtype }\end{array}$ & WGS & N/D & N/D \\
\hline $3719 \mathrm{~T}$ & 74 & M & $\begin{array}{l}\text { Glioblastoma, } \\
\text { IDH-wildtype }\end{array}$ & WGS & N/D & N/D \\
\hline $2211 \mathrm{~T}$ & 75 & $\mathrm{~F}$ & $\begin{array}{l}\text { Glioblastoma, } \\
\text { IDH-wildtype }\end{array}$ & WGS & N/D & N/D \\
\hline $3724 \mathrm{~T}$ & 65 & M & $\begin{array}{l}\text { Glioblastoma, } \\
\text { IDH-wildtype }\end{array}$ & WGS & N/D & N/D \\
\hline
\end{tabular}

Age at initial diagnosis, integrated diagnosis and the type of nanopore sequencing performed are reported. Results of methylation-based random forest classification and key genetic alterations identified by WGS or amplicon sequencing are indicated. Samples were considered not classifiable when there was less than 5 percentage points difference of the majority vote to the next best vote

$W G S$ whole genome sequencing, $N / D$ not done

II denotes false-positive variant

In contrast, focal deletions, such as $C D K N 2 A$, were frequently missed by segmentation. Beyond diagnostic needs, we could reconstruct the double minute nature of an $E G F R$ amplification (case 3427T), identify the exact genomic breakpoint using algorithmic structural variant discovery [36], and confirm the latter by Sanger sequencing (Fig. S2).

\section{Methylation profiling}

A major advantage of nanopore sequencing is the ability to detect base modifications, especially 5-methylation of cytosines, in native DNA without need for bisulfite conversion. Epigenomic changes are functionally important in cancer, but also aid in delineating cancer entities. For example, IDH mutations cause a global hypermethylation of $\mathrm{CpG}$ islands [25], a phenotype of utmost prognostic importance in neuro-oncology. We thus aimed to detect the G-CIMP phenotype from nanopore reads.

First, we compared methylation events in $\mathrm{CpG}$ sites identified by nanopore sequencing to matched methylome microarrays. Good correlation was observed between single read methylation status of a given $\mathrm{CpG}$ site and its corresponding beta value in microarray data (Fig. 2a). Next, we applied random forest (RF) classification to predict IDH mutation.

$\mathrm{RF}$ classification is a commonly used machine-learning algorithm based on randomly generated (weak) decision trees [3]. Majority votes then integrate decisions from the entire forest to provide robust classification. The challenge with low-pass WGS data is that it is not known beforehand which $\mathrm{CpG}$ sites will be sequenced and the classifier can be built upon. Therefore, we generated random forests ad hoc. With increasing numbers of probed $\mathrm{CpG}$ sites, we expect the classifier's error rate to decrease. To test the feasibility of this approach, we simulated multiple random forests for a given number of $\mathrm{CpG}$ sites using the low-grade glioma cohort [5] from The Cancer Genome Atlas (TCGA) and determined misclassification rate for this "random taiga" (Fig. 2b). The simulations show that the mean class error rate to predict IDH and $1 \mathrm{p} / 19 \mathrm{q}$ status does not improve for more than approximately $500 \mathrm{CpG}$ sites. This amount of data is reliably sampled within $6 \mathrm{~h}$ of nanopore sequencing. Thus, information with respect to a cancer's entity is redundantly encoded in the methylome and this fact can be exploited for classification from sparse, randomly sampled CpG sites.

Using the same training set, we then predicted IDH status in our samples from nanopore-based methylation calls. Due to the low read depth (usually $N=1$ ), methylation calls from nanopore WGS were binary. To enable classification using microarray-based training data, beta values were dichotomized as described in previous applications of $\mathrm{RF}$ in methylation data [5, 7]. All samples were correctly classified (Fig. 2c).

\section{Supervised pan-cancer classification}

Next, following the idea of a machine-learning-based molecular classification of tumors to fully recognize molecular entities and rule out interobserver variability [32], we sought to investigate whether nanopore $\mathrm{CN}$ and methylation profiles can be used to classify tumor samples on a pan-cancer level. As a training set for all analyses, we used public microarray-based methylation data from primary brain tumors (adult and pediatric glioblastomas, lower grade gliomas, and medulloblastomas) and tumors that frequently metastasize to the brain (melanoma, breast, lung, bladder, prostate, colon, and clear cell renal carcinoma) [1, 

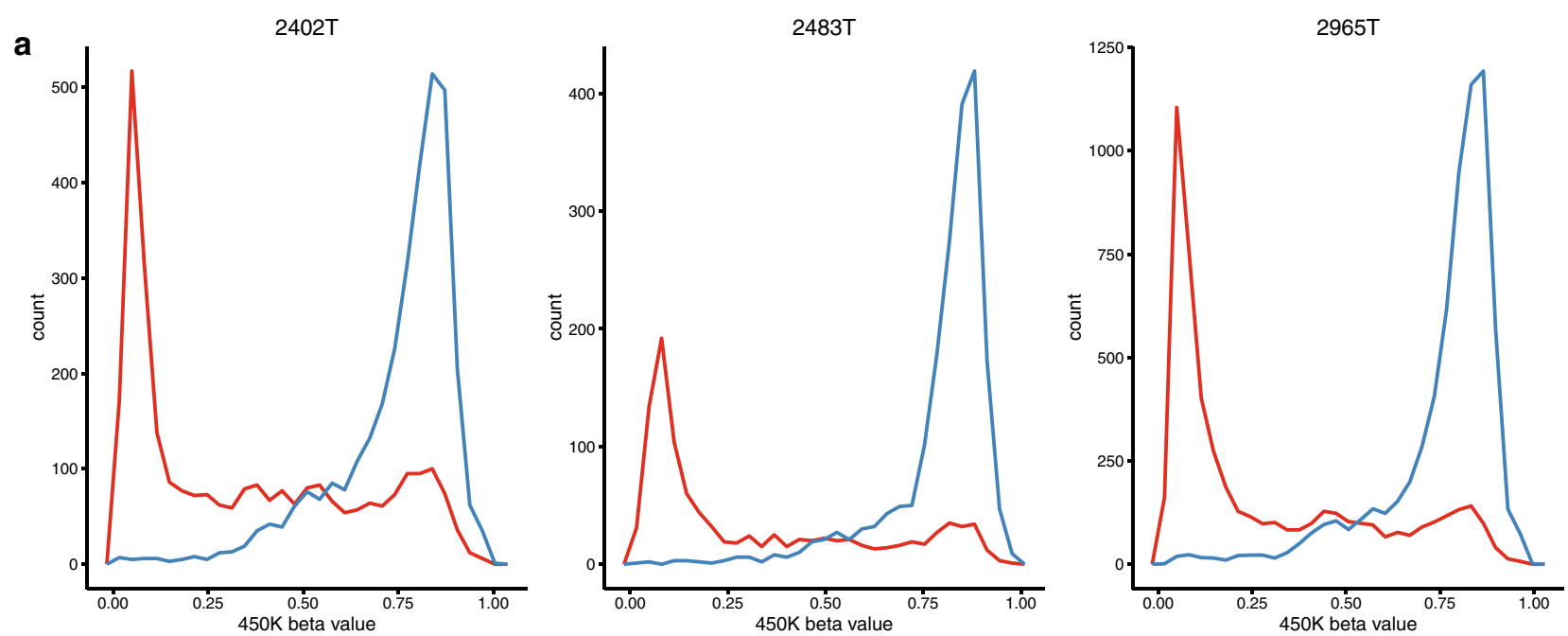

methylated $\mathrm{CpGs}$ unmethylated $\mathrm{CpGs}$
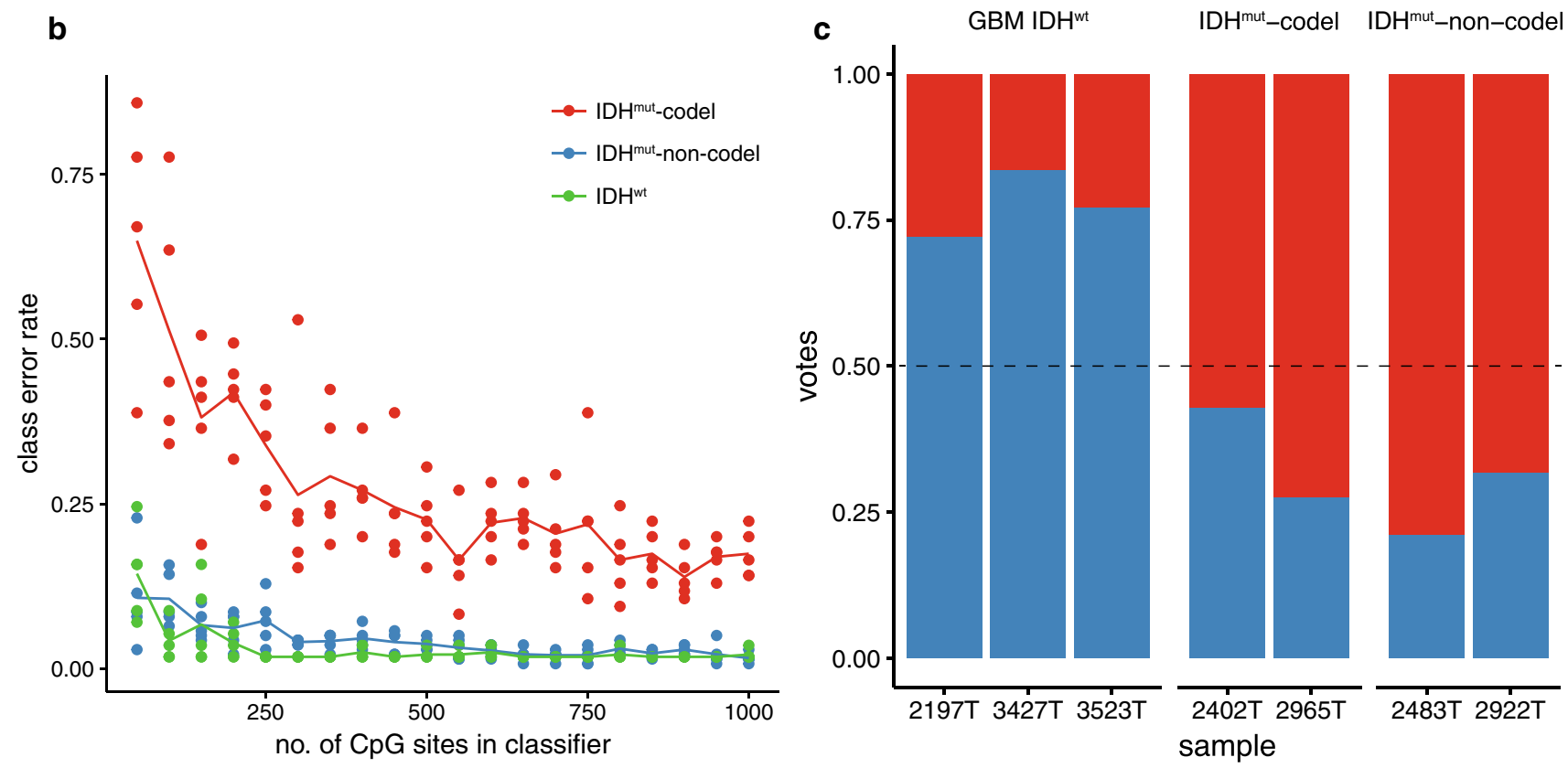

IDH wildtype IDH mutant

Fig. 2 Methylome profiling by nanopore sequencing of native tumor DNA. a Comparison of methylation calls from nanopore sequencing with matched Illumina $450 \mathrm{~K}$ microarray-based data. Beta value distributions for $\mathrm{CpG}$ sites that were identified as unmethylated (red) or methylated (blue), respectively, by nanopore WGS are shown. b "Random taiga" simulation of classification error as a function of the number of randomly sampled $\mathrm{CpG}$ sites. Each dot represents the class-specific error rate of an ad hoc generated random forest using a

2, 4, 5, 12, 23, 24, 37-40]. Where $\mathrm{CN}$ data were available, too, SNP array-based $\mathrm{CN}$ profiles were aggregated to chromosome arm level and added to the training set (Fig. 3a). The resulting classifiers for any set of $\mathrm{CpG}$ sites in our random subset of $N \mathrm{CpG}$ sites (indicated on $X$ axis) from the TCGA lower grade glioma Illumina $450 \mathrm{~K}$ cohort as training set. Lines indicate the mean of five independent simulations. $\mathbf{c}$ Methylation profiles from nanopore sequencing discriminate IDH-mutant and wild-type tumors. Bar plots indicate vote distribution from ad hoc random forest classification. The TCGA low-grade glioma cohort was used as a training set. Illumina 450K-based beta values were dichotomized using $>0.6$ as threshold

cohort usually yielded an overall out-of-bag classification error rate $\ll 5 \%$.

We first subjected seven glioma samples with $\mathrm{CN}$ and methylation profiles generated by nanopore sequencing to 


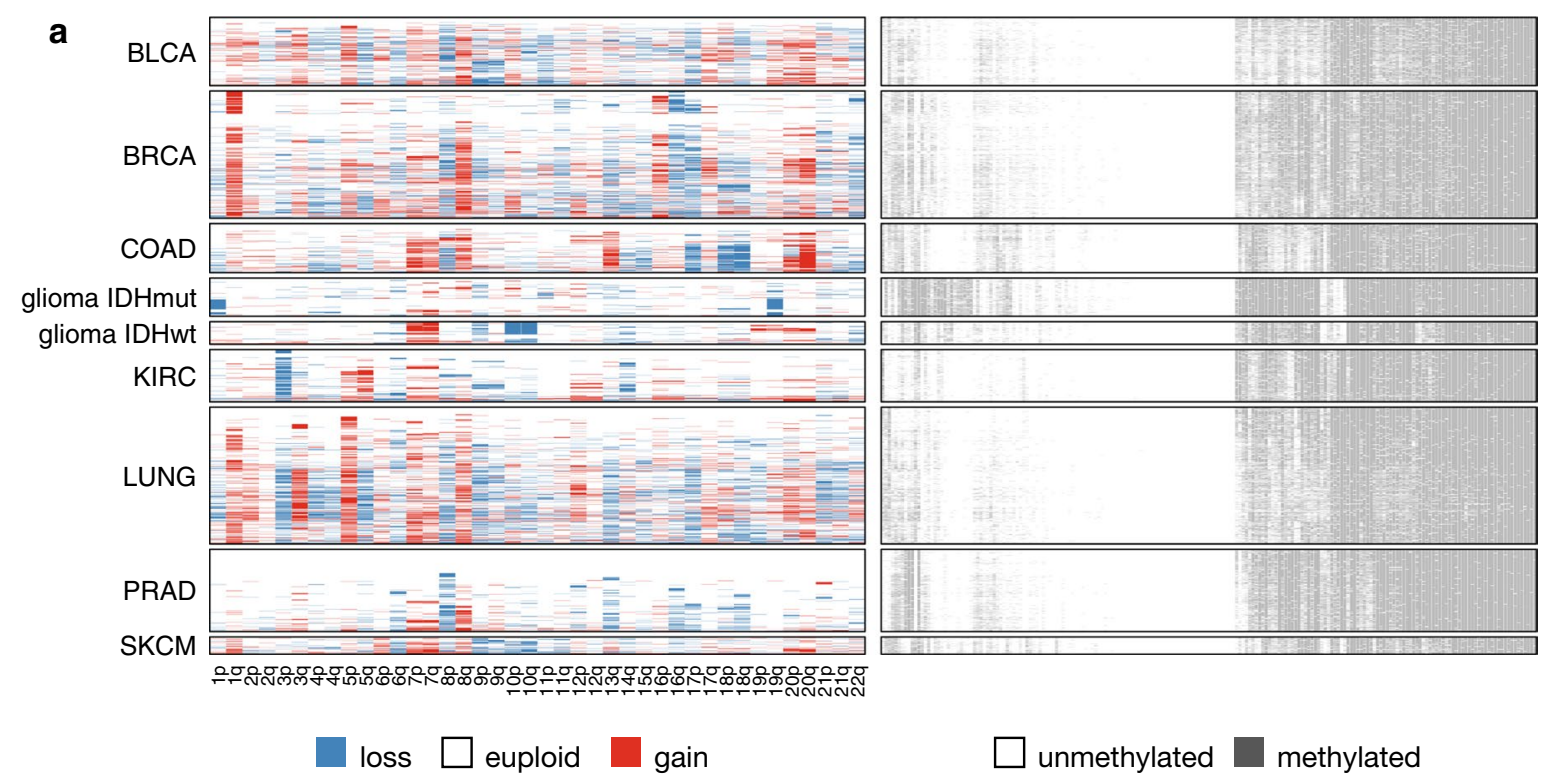

\section{b copy number only}

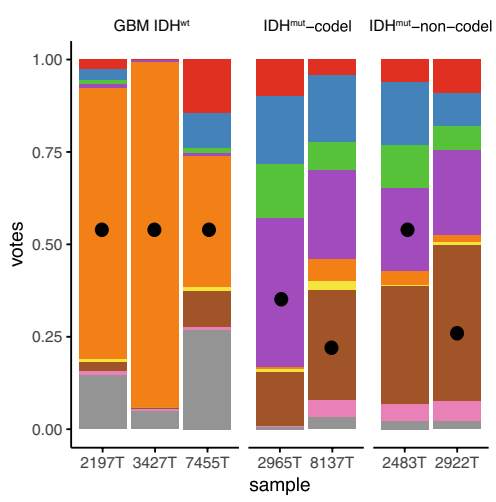

c methylation only

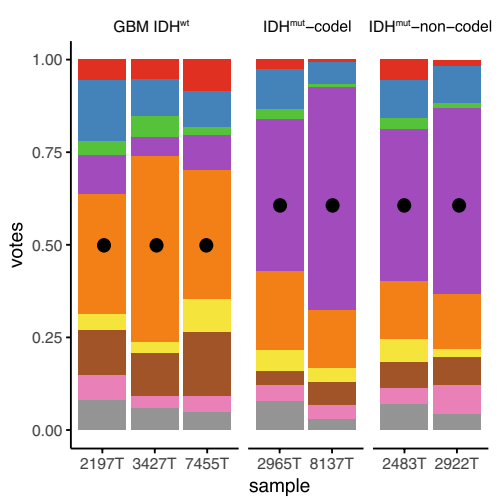

d combined

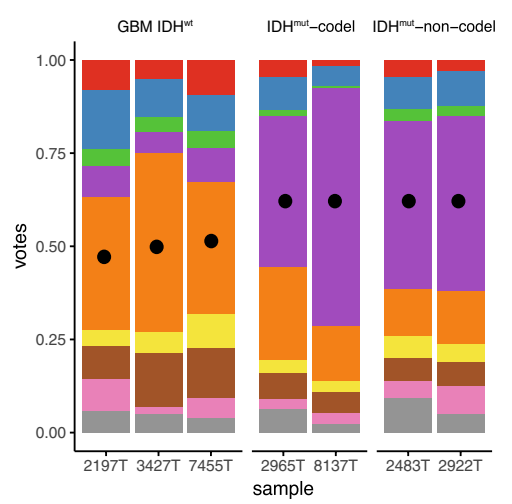

SKCM

PRAD BLCA $\bullet$ majority vote e

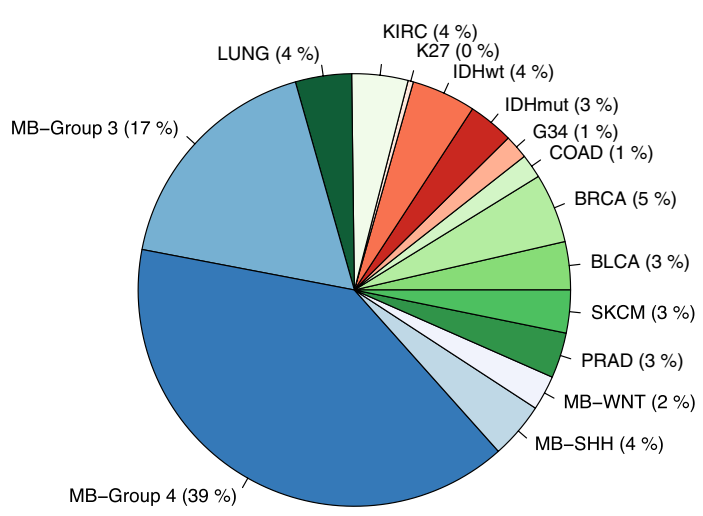

f

8358T

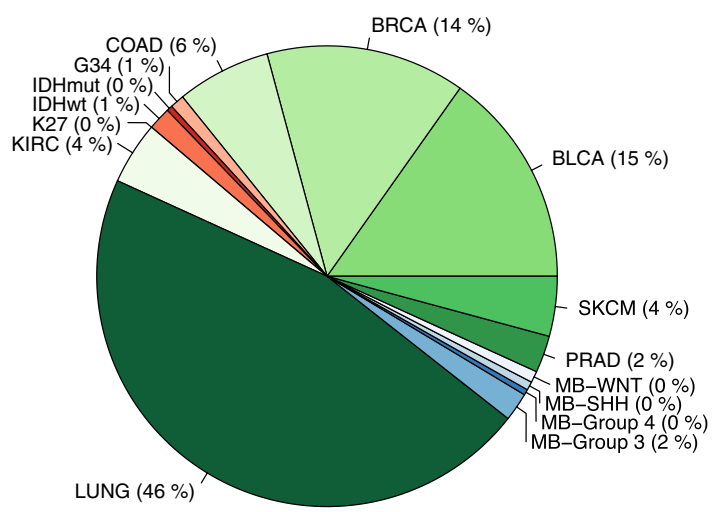


\Fig. 3 Pan-cancer classification using copy number and methylation profiles. a Training set composed of TCGA samples from nine cancer entities using arm-level averaged copy number $(\mathrm{CN})$ information (CN loss blue, $\mathrm{CN}$ gain red) and dichotomized methylation data. For illustration purposes, only 200 random CpG sites were sampled, clustered, and plotted. b-d Classification of samples subjected to WGS using R9.4 flow cells using ad hoc random forests (500 trees per sample). Bar plots show vote distributions based on copy number only (b), methylation (c), or both modalities (d). e, f Methylation-based pan-cancer classification of medulloblastoma (e) and a brain metastasis of a lung adenocarcinoma (f). BRCA breast cancer, $B L C A$ bladder urothelial carcinoma, $C O A D$ colon adenocarcinoma, KIRC kidney renal cell carcinoma, $L U N G$ lung squamous cell and adenocarcinoma, $S K C M$ skin cutaneous melanoma, $P R A D$ prostate adenocarcinoma, $M B$ medulloblastoma, $K 27$ diffuse midline glioma H3 K27M mutant, G34 pediatric glioblastoma, H3 G34R mutant

ad hoc RF classification. When we compared classification using $\mathrm{CN}$ alone (Fig. 3b), methylation only (Fig. 3c) or both modalities together (Fig. 3d), using the joint approach improved overall accuracy: all (7/7) samples were correctly classified.

Then, we subjected two medulloblastoma (MB) cases to classification (here, only methylation training data were available). Both samples were identified as $\mathrm{MB}$ and also the genetic subtype according to the WHO classification was predicted correctly as WNT-activated (case MB683) or non-SSH-activated/non WNT-activated (i.e., group 4, case 8372T) (Fig. 3e). Next, we attempted classification of brain metastasis and could predict the pulmonary origin in one case (Fig. 3f). We also selected a metastasis of a breast adenocarcinoma in the posterior fossa for study which immunohistochemically showed expression of GFAP and S100, so it was misleading for the diagnosis of carcinoma. Pancancer classification based on nanopore WGS correctly identified this sample as breast cancer (Table 1, Fig. S1).

Several cases were not classifiable (requiring a $>5$ percentage points' difference of the majority vote to the next best vote) or misclassified (Table 1). These cases had often lower DNA quality with respect to fragment size (Table S1). One GBM sample that was not classifiable had low tumor purity when estimated from matched transcriptomic profiles using the ESTIMATE algorithm [41] (Fig. S3a). This also resulted in false-negative calling of copy number $\mathrm{CN}$ alterations using fixed thresholds, even though they were present at visual inspection (Fig. S3b).

\section{Amplicon sequencing}

Finally, we explored deep amplicon nanopore sequencing for identification of single nucleotide variants. We designed an amplicon panel covering hotspot exons in $I D H 1, I D H 2$, and $H 3 F 3 A$, all coding exons of TP53 and, additionally, the TERT promoter (pTERT) region. Due to the long reads delivered by nanopore sequencing, this could be achieved with only nine PCR reactions (Table S2). Mutations in these genes (with exception of pTERT) inform molecular diagnosis of glioma and medulloblastoma, and are demanded for diagnosis in the 2016 WHO classification of CNS tumors [20]. Sufficient read depth is a critical parameter for variant calling with defined sensitivity and specificity. We thus implemented a real-time analysis pipeline that allowed monitoring of read depth and to stop sequencing when sufficient information to make a diagnosis has been collected (Fig. 4a). In samples run as single samples with real-time monitoring, a sequencing depth of $1000 \mathrm{X}$ in all target regions could repeatedly be achieved within 2-20 min of sequencing. Mean overall coverage $>1000 X$ could be achieved in single runs, but was lower in runs using barcoding PCR for multiplexing (Fig. 4b).

In all samples, coding mutations were reliably detected as compared to routine diagnostics based on Sanger sequencing, immunohistochemistry or a next-generation sequencing (NGS) panel (Fig. 4c). Nanopore sequencing reads have historically shown high error rates, especially in homopolymer contexts. We, therefore, compared nanopore consensus sequences to matched short-read whole exome data in five cases. Overall concordance was 97.8-98.6\% before functional filtering. Even though at low number ( $<5$ per sample) after filtering for coding mutations, falsepositive variants were present. Most of these mutations occurred in multiple samples, indicating a context-specific error (Table S3). Improved base calling algorithms are thus needed to reduce the time to manually review mutations for false positives.

\section{Technical aspects}

Nanopore sequencing is highly scalable due to low capital cost of the device (use of multiple sequencers) and reuse of flow cells. To exclude carry-over and cross-contamination in sequential sequencing runs and for scalability, we evaluated barcoding and multiplexing for both WGS and amplicon workflows (Table S1, Fig. 4b). For WGS, up to four samples were combined without major protocol changes and permitting convenient overnight runs (e.g., one sample for $6 \mathrm{~h}$ and two samples for $12 \mathrm{~h}$ ). Barcoding of amplicon libraries and multiplexing 12 samples greatly reduces perassay price at the cost of additional PCR and quality control steps. Finally, we explored use of DNA derived from formalin-fixed paraffin-embedded tissue (FFPE). PCR amplicons were generated from two FFPE samples with identical input amount and protocol. As expected from the usually highly fragmented DNA, PCR yields were lower, especially for large amplicons ( $>1 \mathrm{kbp}$ ). This could only partly be compensated by extending sequencing time. For nanopore WGS, transposase-based library preparation is not compatible with fragment size distribution of FFPE-derived 

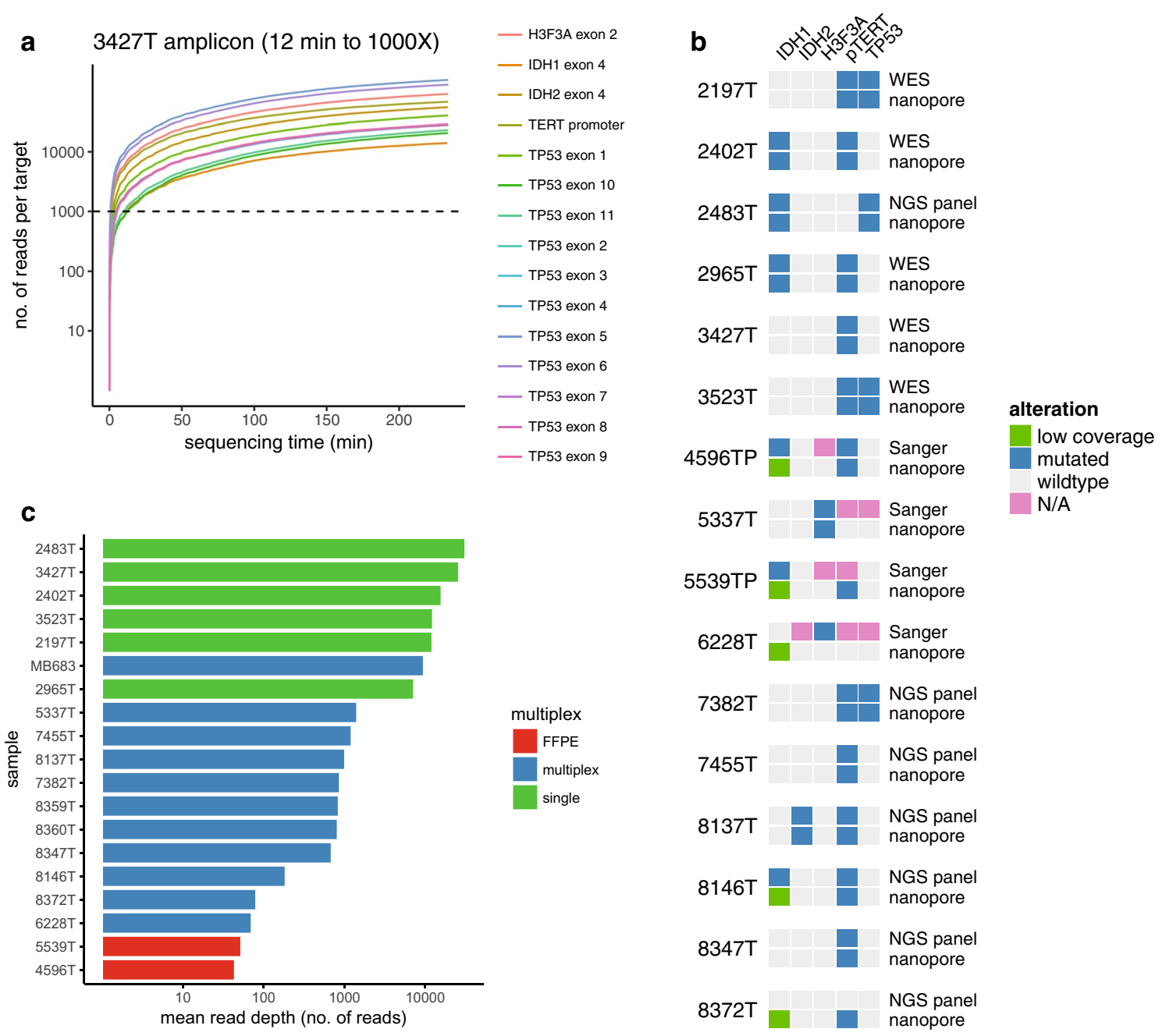

Fig. 4 Real-time amplicon sequencing of single nucleotide variants. a Representative coverage plot of target regions in IDHI, IDH2, H3F3A, TP53, and TERT promoter region over time. The time needed to achieve 1000X depth in all amplicons is indicated. Note log scale on $Y$ axis. b Mean read depth over all amplicons in samples pro-

DNA samples. We thus performed a different ligation protocol to test WGS in one FFPE sample. While read yield was acceptable (Table $\mathrm{S} 1$ ), the resulting copy number profile was noisy and hard to interpret (Fig. S1). In summary, nanopore sequencing is compatible with FFPE samples, but clearly not recommended due to inferior performance.

\section{Discussion}

Histomolecular classification promises to significantly improve diagnosis, prognosis, and treatment decision making of cancer patients by aiding in clearly delineating distinct (molecular) entities and identifying targetable genomic alterations for personalized treatment. It is, therefore, crucial to ensure widespread implementation of cessed individually or as barcoded multiplex libraries. Of note, FFPE samples were sequenced as part of a multiplex library. c Comparison of selected variant calls from nanopore sequencing (filtered for coding or hotspot mutations with minimum allele frequency $>0.2$ ) with reference calls from Sanger or Illumina sequencing

appropriate technology in clinical routine for patient benefit. We explored the potential of nanopore sequencing to comprehensively characterize genetic alterations.

$\mathrm{CN}$ alterations could be detected in brain tumor samples using ultra low-pass WGS. While overall resolution is lower than current SNP arrays or NGS approaches, armlevel alterations and high-level focal alterations are reliably recapitulated. Most importantly, detection of $1 \mathrm{p} / 19 \mathrm{q}$-codeletion fulfills diagnostic needs for the current WHO 2016 classification of CNS tumors. While WGS using rapid, transposase-based library preparation works very well with high molecular weight DNA, some of the clinical routine fresh-frozen tumor DNA samples were highly fragmented and yielded insufficient results. Quality of input DNA thus seems to be pivotal. For use of FFPE material, changes to the protocol and further optimization are needed. 
Methylation data can directly be obtained from the same WGS data set which makes time-consuming bisulfite conversion and specialized methylation assays (sequencing or hybridization-based) expendable. Very recently, it has been shown in the context of meningioma that classification of tumors using methylome data alone is sufficient or superior to make a correct diagnosis [32]. With low genome coverage, we obtained sparse random sampling of $\mathrm{CpG}$ sites. We show that this information is sufficient to subtype gliomas into IDH-mutant vs. wild-type samples and that cancer entities from different tissue origins can be distinguished in a few hours. This may aid in the differential diagnosis of primary brain tumors vs. brain metastases and greatly facilitate staging and the search for unknown primary tumors [22]. However, as diagnosis is inferred from relatively sparse data, it precludes inter-patient comparison and reuse of data with currently obtainable coverage in the (relatively short) time frame of $6 \mathrm{~h}$ of sequencing.

Finally, we used PCR-based amplicon generation followed by nanopore sequencing to identify point mutations. Using a small, but diagnostically relevant gene panel (covering target regions with a total of $12 \mathrm{~kb}$ ), high read depth could be routinely obtained in less than $30 \mathrm{~min}$ of sequencing when using real-time depth monitoring. However, context-specific base calling errors introduce platform-specific errors and false variant calls that need to be carefully reviewed.

\section{Comparison to existing technologies}

Targeted next-generation sequencing panels tailored to detect mutations in brain tumors or, more generally, cancer-related genes have been employed routinely with a turnaround time of several days [8, 31]. Methylation-based classification of brain tumors by microarray allows differentiation of a wealth of different entities within 2 weeks [12, 32]. Intraoperative subtyping of gliomas is possible using allele specific PCR for key alterations (IDH1, pTERT) but remains restricted to hotspot point mutations [34]. Similarly, $\mathrm{CN}$ changes and mutations have been detected in cell-free DNA from CSF to allow less invasive diagnostics [10, 26]. A major drawback of all approaches is the high investment cost, need for laboratory space or expertise.

For nanopore sequencing, besides the portable sequencing device and a laptop computer, only a spectrometer for DNA quantification and a thermocycler for library preparation and amplicon generation by PCR are needed. This allows implementation of a complete molecular pathology laboratory even in resource-restricted settings or mobile environments. Per sample cost is $\sim \$ 200$ for WGS and $\sim \$ 120$ for amplicon sequencing without multiplexing. However, being a technology still under development, frequent updates in chemistry and software currently challenge routine use and need to be addressed to allow standardized diagnostics across laboratories. In addition, hybridization microarrays and targeted shortread sequencing both work relatively well with fragmented DNA from FFPE samples, while this currently poses a technical challenge for nanopore sequencing.

Our study has several limitations. First, as this is a proof-of-principle study, sample number is small and precludes accurate quantification of sensitivity or specificity to detect structural alterations and point mutations. Second, a prospective and multi-centric evaluation of the approach presented here is needed to rule out sample selection bias and demonstrate robustness across laboratories. Third, we reused flow cells to reduce per-assay cost, but washing also decreased the number of active pores and thus performance in subsequent runs.

In conclusion, same-day diagnosis of $\mathrm{CN}$ alterations, epigenetic modifications, and single nucleotide variants using nanopore sequencing is feasible with minimal capital cost and without need for sophisticated laboratory equipment. For CNS tumors, molecular features demanded for diagnosis by current guidelines can be obtained, which, together with histological data and grading, enable accelerated integrated diagnosis and improve patient care.

Acknowledgements The authors would like to acknowledge Jared T. Simpson for providing methylation training models, Inès Detrait and Amithys Rahimian-Aghda for biobanking and sample management, Ludovic Prevost for excellent IT systems administration, and Mark van Roosmalen for assistance with running NanoSV. We are indebted to the Nanopore Human Genome Sequencing consortium for early release of data at https://github.com/nanopore-wgs-consortium/ NA12878. The results published here are in part based upon data generated by The Cancer Genome Atlas (TCGA) project established by the NCI and NHGRI. Information about TCGA and the investigators and institutions who constitute the TCGA research network can be found at http://cancergenome.nih.gov/.

\section{Compliance with ethical standards}

Funding This work has been supported by Deutsche Forschungsgemeinschaft (EU 162/1-1 to PE), the program "Investissements d'avenir" (ANR-10-IAIHU-06 to AI), Institut Universitaire de Cancérologie (to AI), Ligue Nationale Contre le Cancer (to AI), Institut Carnot (to KL), and Fondation ARC pour la recherche sur le cancer (nPJA 20151203562 to FB).

Conflict of interest The authors declare no conflicts of interest.

Open Access This article is distributed under the terms of the Creative Commons Attribution 4.0 International License (http://creativecommons.org/licenses/by/4.0/), which permits unrestricted use, distribution, and reproduction in any medium, provided you give appropriate credit to the original author(s) and the source, provide a link to the Creative Commons license, and indicate if changes were made. 


\section{References}

1. Abeshouse A, Ahn J, Akbani R, Ally A, Amin S, Andry CD et al (2015) The molecular taxonomy of primary prostate cancer. Cell 163:1011-1025. doi:10.1016/j.cell.2015.10.025

2. Akbani R, Akdemir KC, Aksoy BA, Albert M, Ally A, Amin $\mathrm{SB}$ et al (2015) Genomic classification of cutaneous melanoma. Cell 161:1681-1696. doi:10.1016/j.cell.2015.05.044

3. Breiman L (2001) Random forests. Mach Learn 45:5-32. doi:1 0.1023/A:1010933404324

4. Brennan CW, Verhaak RGW, McKenna A, Campos B, Noushmehr H, Salama SR et al (2013) The somatic genomic landscape of glioblastoma. Cell 155:462-477. doi:10.1016/j. cell.2013.09.034

5. Brat DJ, Verhaak RGW, Aldape KD, Yung WKA, Salama SR, Cancer Genome Atlas Research Network et al (2015) Comprehensive, integrative genomic analysis of diffuse lowergrade gliomas. N Engl J Med 372:2481-2498. doi:10.1056/ NEJMoa1402121

6. Cao MD, Ganesamoorthy D, Cooper MA, Coin LJM (2016) Realtime analysis and visualization of MinION sequencing data with npReader. Bioinformatics 32:764-766. doi:10.1093/ bioinformatics/btv658

7. Ceccarelli M, Barthel FP, Malta TM, Sabedot TS, Salama SR, Murray BA et al (2016) Molecular profiling reveals biologically discrete subsets and pathways of progression in diffuse glioma. Cell 164:550-563. doi:10.1016/j.cell.2015.12.028

8. Cheng DT, Mitchell TN, Zehir A, Shah RH, Benayed R, Syed A et al (2015) Memorial Sloan Kettering-Integrated Mutation Profiling of Actionable Cancer Targets (MSK-IMPACT): a hybridization capture-based next-generation sequencing clinical assay for solid tumor molecular oncology. J Mol Diagn 17:251-264. doi:10.1016/j.jmoldx.2014.12.006

9. Cingolani P, Platts A, Wang LL, Coon M, Nguyen T, Wang L et al (2012) A program for annotating and predicting the effects of single nucleotide polymorphisms, SnpEff: SNPs in the genome of Drosophila melanogaster strain w1118; iso-2; iso-3. Fly (Austin) 6:80-92. doi:10.4161/fly.19695

10. De Mattos-Arruda L, Mayor R, Ng CKY, Weigelt B, MartínezRicarte F, Torrejon D et al (2015) Cerebrospinal fluid-derived circulating tumour DNA better represents the genomic alterations of brain tumours than plasma. Nat Commun 6:8839. doi:10.1038/ncomms9839

11. Goldman M, Craft B, Swatloski T, Ellrott K, Cline M, Diekhans M et al (2013) The UCSC cancer genomics browser: update 2013. Nucl Acids Res 41:D949-D954. doi:10.1093/nar/ gks 1008

12. Hovestadt V, Remke M, Kool M, Pietsch T, Northcott PA, Fischer R et al (2013) Robust molecular subgrouping and copy-number profiling of medulloblastoma from small amounts of archival tumour material using high-density DNA methylation arrays. Acta Neuropathol 125:913-916. doi:10.1007/s00401-013-1126-5

13. Huber W, Carey VJ, Gentleman R, Anders S, Carlson M, Carvalho BS et al (2015) Orchestrating high-throughput genomic analysis with bioconductor. Nat Methods 12:115-121. doi:10.1038/nmeth.3252

14. Kamoun A, Idbaih A, Dehais C, Elarouci N, Carpentier C, Letouzé $E$ et al (2016) Integrated multi-omics analysis of oligodendroglial tumours identifies three subgroups of $1 \mathrm{p} / 19 \mathrm{q}$ co-deleted gliomas. Nat Commun 7:11263. doi:10.1038/ ncomms 11263

15. Koboldt DC, Zhang Q, Larson DE, Shen D, McLellan MD, Lin L et al (2012) VarScan 2: somatic mutation and copy number alteration discovery in cancer by exome sequencing. Genome Res 22:568-576. doi:10.1101/gr.129684.111
16. Lek M, Karczewski KJ, Minikel EV, Samocha KE, Banks E, Fennell T et al (2016) Analysis of protein-coding genetic variation in 60,706 humans. Nature 536:285-291. doi:10.1038/ nature 19057

17. Li H, Durbin R (2009) Fast and accurate short read alignment with Burrows-Wheeler transform. Bioinformatics 25:17541760. doi:10.1093/bioinformatics/btp324

18. Loman NJ, Quinlan AR (2014) Poretools: a toolkit for analyzing nanopore sequence data. Bioinformatics 30:3399-3401. doi:10.1093/bioinformatics/btu555

19. Loose M, Malla S, Stout M (2016) Real-time selective sequencing using nanopore technology. Nat Methods. doi:10.1038/nmeth.3930

20. Louis DN, Perry A, Reifenberger G, von Deimling A, Figarella-Branger D, Cavenee WK et al (2016) The 2016 World Health Organization classification of tumors of the central nervous system: a summary. Acta Neuropathol 131:803-820. doi:10.1007/s00401-016-1545-1

21. McKenna A, Hanna M, Banks E, Sivachenko A, Cibulskis K, Kernytsky A et al (2010) The genome analysis toolkit: a MapReduce framework for analyzing next-generation DNA sequencing data. Genome Res 20:1297-1303. doi:10.1101/gr.107524.110

22. Moran S, Martínez-Cardús A, Sayols S, Musulén E, Balañá C, Estival-Gonzalez A et al (2016) Epigenetic profiling to classify cancer of unknown primary: a multicentre, retrospective analysis. Lancet Oncol 17:1386-1395. doi:10.1016/ S1470-2045(16)30297-2

23. Network TCGA (2012) Comprehensive molecular characterization of human colon and rectal cancer. Nature 487:330-337. doi:10.1038/nature11252

24. Network TCGAR (2012) Comprehensive genomic characterization of squamous cell lung cancers. Nature 489:519-525. doi:10.1038/nature11404

25. Noushmehr H, Weisenberger DJ, Diefes K, Phillips HS, Pujara $\mathrm{K}$, Berman BP et al (2010) Identification of a CpG island methylator phenotype that defines a distinct subgroup of glioma. Cancer Cell 17:510-522. doi:10.1016/j.ccr.2010.03.017

26. Pentsova EI, Shah RH, Tang J, Boire A, You D, Briggs S et al (2016) Evaluating cancer of the central nervous system through next-generation sequencing of cerebrospinal fluid. J Clin Oncol. doi:10.1200/JCO.2016.66.6487

27. Quick J, Loman NJ, Duraffour S, Simpson JT, Severi E, Cowley L et al (2016) Real-time, portable genome sequencing for Ebola surveillance. Nature 530:228-232. doi:10.1038/nature16996

28. Quinlan AR, Hall IM (2010) BEDTools: a flexible suite of utilities for comparing genomic features. Bioinformatics 26:841842. doi:10.1093/bioinformatics/btq033

29. Rand AC, Jain M, Eizenga JM, Musselman-Brown A, Olsen HE, Akeson $M$ et al (2017) Mapping DNA methylation with highthroughput nanopore sequencing. Nat Methods. doi:10.1038/ nmeth.4189

30. Rosenberg S, Verreault M, Schmitt C, Guegan J, Guehennec J, Levasseur C et al (2017) Multi-omics analysis of primary glioblastoma cell lines shows recapitulation of pivotal molecular features of parental tumors. Neuro-Oncol 19:219-228. doi:10.1093/ neuonc/now 160

31. Sahm F, Schrimpf D, Jones DTW, Meyer J, Kratz A, Reuss D et al (2016) Next-generation sequencing in routine brain tumor diagnostics enables an integrated diagnosis and identifies actionable targets. Acta Neuropathol 131:903-910. doi:10.1007/ s00401-015-1519-8

32. Sahm F, Schrimpf D, Stichel D, Jones DTW, Hielscher T, Schefzyk S et al (2017) DNA methylation-based classification and grading system for meningioma: a multicentre, retrospective analysis. Lancet Oncol. doi:10.1016/S1470-2045(17)30155-9 
33. Scheinin I, Sie D, Bengtsson H, van de Wiel MA, Olshen $\mathrm{AB}$, van Thuijl HF et al (2014) DNA copy number analysis of fresh and formalin-fixed specimens by shallow whole-genome sequencing with identification and exclusion of problematic regions in the genome assembly. Genome Res 24:2022-2032. doi:10.1101/gr.175141.114

34. Shankar GM, Francis JM, Rinne ML et al (2015) Rapid intraoperative molecular characterization of glioma. JAMA Oncol. doi:10.1001/jamaoncol.2015.0917

35. Simpson JT, Workman RE, Zuzarte PC, David M, Dursi LJ, Timp W (2017) Detecting DNA cytosine methylation using nanopore sequencing. Nat Methods. doi:10.1038/nmeth.4184

36. Stancu MC, Roosmalen MJ van, Renkens I, Nieboer M, Middelkamp S, Ligt J de, et al. (2017) Mapping and phasing of structural variation in patient genomes using nanopore sequencing. bioRxiv 129379. doi:10.1101/129379

37. Sturm D, Witt H, Hovestadt V, Khuong-Quang D-A, Jones DTW, Konermann C et al (2012) Hotspot mutations in H3F3A and IDH1 define distinct epigenetic and biological subgroups of glioblastoma. Cancer Cell 22:425-437. doi:10.1016/j. ccr.2012.08.024

38. The Cancer Genome Atlas Research Network (2013) Comprehensive molecular characterization of clear cell renal cell carcinoma. Nature 499:43-49. doi:10.1038/nature 12222

39. The Cancer Genome Atlas Research Network (2014) Comprehensive molecular profiling of lung adenocarcinoma. Nature 511:543-550. doi:10.1038/nature13385

40. The Cancer Genome Atlas Research Network (2014) Comprehensive molecular characterization of urothelial bladder carcinoma. Nature 507:315-322. doi:10.1038/nature12965

41. Yoshihara K, Shahmoradgoli M, Martínez E, Vegesna R, Kim H, Torres-Garcia W et al (2013) Inferring tumour purity and stromal and immune cell admixture from expression data. Nat Commun 4:2612. doi:10.1038/ncomms3612 